\title{
Analytical Solutions of Excited Vibrations of a Beam with Application of Distribution
}

\author{
M.S. KOZIENं* \\ Institute of Applied Mechanics, Cracow University of Technology \\ al. Jana Pawła II 37, 31-864 Kraków, Poland
}

\begin{abstract}
In the paper, the analytical solutions of excited vibrations of the Bernoulli-Euler type beam in general case of external loading function is analyzed. The distribution theory is applied to formulate solution when the external functions are the concentrated-force type or the concentrated-moment type. Moreover, two types of excitation in time domain, harmonic and pulsed, are considered. Due to the superposition rule which can be applied in the analyzed linear case, any combination of external loading function can be formulated. The strict analytical solutions are shown for the case of simply supported beam. Describing the external load in the form of concentrated moments makes possible the analytical simulation of the reduction of vibrations of a beam by application of the piezoelectric elements which are in practice the source of external moment-type excitation put in relatively small area of action.
\end{abstract}

DOI: 10.12693/APhysPolA.123.1029

PACS: $02.30 . J r$, 43.40.Ar, 43.40.Cw, 45.20.da

\section{Introduction}

The natural way for describing the external excitation in equation of motion of the Bernoulli-Euler type beam is putting the loading function in a form of linearly distributed force $q(x, t)$. In realistic application sometimes is better to model the external force in a form of set of concentrated forces $F_{i}\left(x_{i}, t\right)$, or set of concentrated moments $M_{j}\left(x_{j}, t\right)$. From mathematical point of view, the concentrated force and moment can be put in the beam equation of motion with application of the distributions. The most popular distribution used in the theory of vibrations is the Heaviside distribution $\Theta(x)$ [1]. In application it makes possible to describe the step-type of material or geometrical parameters of beams. In the case of smart beams damped by distributed piezoelectric elements [2] glued to the external surfaces of beam, the position of each of the elements can be described by suitable pair of the Heaviside distribution $\Theta\left(x-x_{1}\right)$ and $\Theta\left(x-x_{2}\right)$. The examples of application for theoretical formulation of the problem can be found in [3-5]. The other popular distribution is the Dirac delta distribution $\delta(x)$ [1]. The examples of its application to describe the position of the concentrated force action is given in [6-9] for beams or in $[9,10]$ for plates. The place of action and action of concentrated moment can be described by the first derivative of the Dirac delta distribution $\delta^{\prime}(x)$ [1]. The concentrated moments can be used as the model of action of piezoelectric distributed elements $[4,11,12]$.

In the paper [13], the mathematical problem of analytical solution of vibration of plates with piezoelectric actuators with arbitrary shape in distribution formulation is

*e-mail: kozien@mech.pk.edu.pl discussed. In [10], a group of general analytical solutions for externally excited plates with different boundary conditions (understood as given eigenfunctions) for different types of concentrated forces and distributed forces are given.

In the presented paper, a group of general analytical solutions for externally excited beams with different boundary conditions (understood as given eigenfunctions) for different types of concentrated force, distributed moment, and distributed force is given. The given solutions can be applied to analyze of transient vibrations of simple realistic structures, as a test cases for advanced models and for the analysis of the control process with application of piezoelectric elements modeled by concentrated moments.

\section{Analytical solution}

\subsection{Formulation of the problem}

Let us consider the problem of excited vibrations of the Bernoulli-Euler beam with arbitrary boundary conditions

$$
\left\{\begin{array}{l}
E I \frac{\partial^{4} w(x, t)}{\partial x^{4}}+\rho A \frac{\partial^{2} w(x, t)}{\partial t^{2}}=f(x, t) \\
f(x, t)=q(x, t)+\sum_{i=1}^{N} F_{i} \delta\left(x-x_{i}\right) \\
\quad+\sum_{j=1}^{M} M_{j} \frac{\mathrm{d}}{\mathrm{d} x} \delta\left(x-x_{j}\right)
\end{array},\right.
$$

where $w(x, t)$ is the transverse displacement of a beam, $E$ is the Young modulus of material, $\rho$ is the volume density of material, $I$ is the moment of inertia of the beam cross-section, $A$ is the area of the beam cross-section, $M_{j}(t)$ is the j-th moment, $x_{j}$ is the point of action of $j$ th moment, $F_{i}(t)$ is the $\mathrm{i}$-th concentrated force, $x_{i}$ is the point of action of $i$-th concentrated force, $q(x, t)$ is the distributed force. 
Solution of the problem is a sum of the general solution of the homogeneous differential Eq. (2) connected with general Eq. (1) - natural vibrations, and a special solution of the non-homogeneous Eq. (1) - excited vibrations.

\subsection{The eigen-problem}

Let us consider non-excited vibrations of a BernoulliEuler beam with length $l$. Equation of motion has the form

$$
E I \frac{\partial^{4} w(x, t)}{\partial x^{4}}+\rho A \frac{\partial^{2} w(x, t)}{\partial t^{2}}=0 .
$$

Equation of motion should be fulfilled by initial conditions (3) and the boundary conditions (4), where $w_{0}(x)$ is the initial displacement of the beam for $t=0$, and $v_{0}(x)$ is the initial transversal velocity of the beam for $t=0$ :

$$
\begin{aligned}
& \left\{\begin{array}{l}
w(x, 0)=w_{0}(x) \\
\frac{\partial}{\partial t} w(x, 0)=v_{0}(x)
\end{array}\right. \\
& \left\{\begin{array}{l}
w(0, t)=0 \text { or } E I \frac{\partial^{3}}{\partial x^{3}} w(0, t)=0 \\
\frac{\partial}{\partial x} w(0, t)=0 \text { or } E I \frac{\partial^{2}}{\partial x^{2}} w(0, t)=0 \\
w(l, t)=0 \text { or } E I \frac{\partial^{3}}{\partial x^{3}} w(l, t)=0 \\
\frac{\partial}{\partial x} w(l, t)=0 \text { or } E I \frac{\partial^{2}}{\partial x^{2}} w(l, t)=0
\end{array}\right.
\end{aligned}
$$

Solution of the problem is proposed in the form (5) the Fourier method of solution. After suitable manipulations, it leads solution to the problem of separated

variables (6). The form of unknown functions in spatial domain can be written in the form (7). For given homogeneous boundary conditions (4), these series of functions satisfy the orthogonality conditions (8). Finally, the solution can be written in the form (9), where series of constants $a_{n}$ and $b_{n}$ are determined based on the initial conditions (3):

$$
\begin{aligned}
& w(x, t)=\sum_{n=1}^{+\infty} X_{n}(x) T_{n}(t), \\
& \frac{\frac{d^{4} X_{n}(x)}{\mathrm{d} x^{4}}}{X_{n}(x)}=-\frac{\rho A}{E I} \frac{\frac{d^{2} T_{n}(t)}{\mathrm{d} t^{2}}}{T_{n}(t)}=\lambda_{n}^{4}, \\
& \left\{\begin{array}{l}
X_{n}(x)=A_{n} \sin \left(\lambda_{n} x\right)+B_{n} \cos \left(\lambda_{n} x\right)+ \\
C_{n} \operatorname{sh}\left(\lambda_{n} x\right)+D_{n} \operatorname{ch}\left(\lambda_{n} x\right) \\
\omega_{n}=\lambda_{n}^{2} \sqrt{\frac{E I}{\rho A}}
\end{array}\right. \\
& \left\{\begin{array}{l}
1 \\
X_{n}(x) X_{m}(x) \mathrm{d} x=\left\{\begin{array}{l}
\gamma_{n}^{2} \\
0
\end{array} \quad n \neq m\right.
\end{array},\right. \\
& \left\{\begin{array}{l}
w(x, t)=\sum_{n=1}^{+\infty} X_{n}(x)\left[a_{n} \sin \left(\omega_{n} t\right)+b_{n} \cos \left(\omega_{n} t\right)\right] \\
a_{n}=\frac{1}{\omega_{n} \gamma_{n}^{2}} \int_{0}^{l} v_{0}(x) X_{n}(x) \mathrm{d} x . \\
b_{n}=\frac{1}{\gamma_{n}^{2}} \int_{0}^{1} w_{0}(x) X_{n}(x) \mathrm{d} x
\end{array}\right.
\end{aligned}
$$

\section{TABLE I}

Values of parameters $\lambda_{n}$ and approximate formulae (after [14, 15])

\begin{tabular}{c|c|c|c|c}
\hline \hline Boundary conditions & $\lambda_{1} l$ & $\lambda_{2} l$ & $\lambda_{n} l$, large $n$ & Formula \\
\hline SS-SS & $\pi$ & $2 \pi$ & $n \pi$ (exact) & $\sin \left(\lambda_{n} x\right)=0$ \\
\hline FR-FR & 4.7300 & 7.8532 & $\frac{2 n+1}{2} \pi$ & $\cos \left(\lambda_{n} x\right) \operatorname{ch}\left(\lambda_{n} x\right)=1$ \\
\hline FX-FX & 4.7300 & 7.8532 & $\frac{2 n+1}{2} \pi$ & $\cos \left(\lambda_{n} x\right) \operatorname{ch}\left(\lambda_{n} x\right)=1$ \\
\hline FX-SS & 3.9266 & 7.0686 & $\frac{4 n+1}{4} \pi$ & $\tan \left(\lambda_{n} x\right)=\operatorname{th}\left(\lambda_{n} x\right)$ \\
\hline FX-FR & 1.8751 & 4.6941 & $\frac{2 n-1}{2} \pi$ & $\cos \left(\lambda_{n} x\right) \operatorname{ch}\left(\lambda_{n} x\right)=-1$
\end{tabular}

TABLE II

Eigenfunctions for selected boundary conditions of beam (after [14, 15])

\begin{tabular}{c|c}
\hline \hline Boundary conditions & $X_{n}(x)$ \\
\hline SS-SS & $\sin \left(\lambda_{n} x\right)$ \\
\hline FR-FR & {$\left[\operatorname{ch}\left(\lambda_{n} x\right)-\cos \left(\lambda_{n} x\right)\right]\left[\operatorname{ch}\left(\lambda_{n} x\right)+\cos \left(\lambda_{n} x\right)\right]-\left[\operatorname{sh}\left(\lambda_{n} x\right)+\sin \left(\lambda_{n} x\right)\right]\left[\operatorname{sh}\left(\lambda_{n} x\right)+\sin \left(\lambda_{n} x\right)\right]$} \\
\hline FX-FX & {$\left[\operatorname{ch}\left(\lambda_{n} x\right)-\cos \left(\lambda_{n} x\right)\right]\left[\operatorname{ch}\left(\lambda_{n} x\right)-\cos \left(\lambda_{n} x\right)\right]-\left[\operatorname{sh}\left(\lambda_{n} x\right)+\sin \left(\lambda_{n} x\right)\right]\left[\operatorname{sh}\left(\lambda_{n} x\right)+\sin \left(\lambda_{n} x\right)\right]$} \\
\hline FX-SS & {$\left[\operatorname{sh}\left(\lambda_{n} x\right)-\sin \left(\lambda_{n} x\right)\right]\left[\operatorname{ch}\left(\lambda_{n} x\right)-\cos \left(\lambda_{n} x\right)\right]-\left[\operatorname{ch}\left(\lambda_{n} x\right)-\cos \left(\lambda_{n} x\right)\right]\left[\operatorname{sh}\left(\lambda_{n} x\right)-\sin \left(\lambda_{n} x\right)\right]$} \\
\hline FX-FR & {$\left[\operatorname{sh}\left(\lambda_{n} x\right)+\sin \left(\lambda_{n} x\right)\right]\left[\operatorname{ch}\left(\lambda_{n} x\right)-\cos \left(\lambda_{n} x\right)\right]-\left[\operatorname{ch}\left(\lambda_{n} x\right)+\cos \left(\lambda_{n} x\right)\right]\left[\operatorname{sh}\left(\lambda_{n} x\right)-\sin \left(\lambda_{n} x\right)\right]$}
\end{tabular}

Finding the solution of the differential Eq. (2) for a given boundary conditions (3) is the well-known eigenmode problem which gives a set of eigen-values (powered natural frequencies of a system $\omega_{n}^{2}$ ) and a set of eigenfunctions (eigen-modes, waveforms of eigen-functions $\left.X_{n}(x)\right)$. The values of parameters $\lambda_{n}$ which makes pos- 
sible to find natural frequencies (7) for different combination of typical types of boundary conditions (simply supported, free, fixed) are given in Table I. The analytical form of eigen-functions for the same combination of boundary conditions (simply supported, free, fixed) is given in Table II. The other name of the problem is the modal problem. After taking into account the initial values, the solution (9), representing free vibrations, which is the response of the system to initial conditions written in time and spatial domains $w(x, t)$, can be obtained.

\subsection{Excited vibrations}

When analyzing the excited vibrations of realistic beam (with internal damping), the general solution of the homogeneous differential equation is a function which relatively fast tending to zero with respect to time due to internal and external damping.

The full solution of the problem of excited vibrations, understood as the special solution of the nonhomogeneous Eq. (1), has for excitation function of harmonic in time domain type, the form of sum of two components: connected with a set of natural frequencies $\omega_{n}$, and connected with external loading frequency $\nu$. Due to the same result of action of internal and external damping (as for natural vibrations), the components connected with set of natural frequencies $\omega_{n}$ (free vibrations) are functions relatively fast tending to zero with respect to time. Therefore, the solution of exited vibrations is usually those, connected with the only external loading frequency $\nu[8,9,14]$. Such case of vibrations is called as steady-state case. The complete solution of the problem which includes free vibrations and excited vibrations in general formulation, is called as transient vibrations.

Due to application discussed in Introduction, let us consider the general form of solution of excited vibrations. For such a case, the solution can be proposed in the form (10) in terms of the functions $X_{n}(x)$ and series of unknown functions of time $H_{n}(t)$. The external load function existing in Eq. (1) can be represented in series form (11), in terms of functions $X_{n}(x)$ and series of known functions of time $Q_{n}(t)$ :

$$
\begin{aligned}
& w(x, t)=\sum_{n=1}^{+\infty} X_{n}(x) H_{n}(t), \\
& \left\{\begin{array}{l}
f(x, t)=\sum_{n=1}^{+\infty} X_{n}(x) Q_{n}(t) \\
Q_{n}(t)=\frac{1}{\rho A} \frac{1}{\gamma_{n}^{2}} \int_{0}^{1} f(x, t) X_{n}(x) \mathrm{d} x
\end{array} .\right.
\end{aligned}
$$

Substituting the formulae (10) and (11) into Eq. (1), the differential equation for determination of unknown functions $H_{n}(t)$ takes the form (12)

$$
\frac{d^{2} H_{n}(t)}{\mathrm{d} t^{2}}+\omega_{n}^{2} H_{n}(t)=Q_{n}(t) .
$$

Solution of this equation has the form

$$
H_{n}(t)=\frac{1}{\omega_{n}} \int_{0}^{t} Q_{n}(\tau) \sin \left[\omega_{n}(t-\tau)\right] \mathrm{d} \tau,
$$

known as Duhamel integral.
The given formulae make possible to formulate the analytical solution of excited vibrations of a beam with defined boundary conditions.

\section{Formulation of solution of excited vibration of beam by application of distribution}

\subsection{Formulation of the problem}

Let us consider excited vibrations of a beam with given eigenfunctions $X_{n}(x)$. In the following, the cases when external loads are described by the Dirac delta distribution (concentated harmonic force) and its first derivative (concentrated harmonic moment) are analyzed. For each case of a load there are given: form of the external loads $(f(x, t))$, solution in the time domain $\left(H_{n}(t)\right)$, and the complete solution function of displacements $w(x, t)$.

Due to the superposition rule which can be applied in the analyzed linear case, any combination of external load function can be built. Some cases are:

- harmonic concentrated force with constant amplitude applied at a point $x=c$

$$
\begin{aligned}
& f(x, t)=F_{i} \delta(x-c) \sin (\nu t) \\
& H_{n}(t)=\frac{1}{\rho A} F_{i} \frac{1}{\gamma_{n}^{2}} \frac{1}{\omega_{n}^{2}-\nu^{2}} \\
& \quad \times\left[\sin (\nu t)-\frac{\nu}{\omega_{n}} \sin \left(\omega_{n} t\right)\right] X_{n}(c) \\
& w(x, t)=\frac{1}{\rho A} F_{i} \sum_{n=1}^{\infty} \frac{1}{\gamma_{n}^{2}} \frac{1}{\omega_{n}^{2}-\nu^{2}} \\
& \quad \times\left[\sin (\nu t)-\frac{\nu^{2}}{\omega_{n}} \sin \left(\omega_{n} t\right)\right] X_{n}(c) X_{n}(x)
\end{aligned}
$$

- harmonic concentrated moment with constant amplitude applied at a point $x=d$

$$
\begin{aligned}
& f(x, t)=M_{j} \delta^{\prime}(x-d) \sin (\nu t), \\
& H_{n}(t)=-\frac{1}{\rho A} M_{j} \frac{1}{\gamma_{n}^{2}} \frac{1}{\omega_{n}^{2}-\nu^{2}} \\
& \quad \times\left[\sin (\nu t)-\frac{\nu}{\omega_{n}} \sin \left(\omega_{n} t\right)\right] \frac{\mathrm{d}}{\mathrm{d} x} X_{n}(d), \\
& w(x, t)=-\frac{1}{\rho A} M_{j} \sum_{n=1}^{\infty} \frac{1}{\gamma_{n}^{2}} \frac{1}{\omega_{n}^{2}-\nu^{2}} \\
& \quad \times\left[\sin (\nu t)-\frac{\nu}{\omega_{n}} \sin \left(\omega_{n} t\right)\right] \frac{\mathrm{d}}{\mathrm{d} x} X_{n}(d) X_{n}(x)
\end{aligned}
$$

- pulsed concentrated force with constant amplitude acting at time $t_{0}$

$$
\begin{aligned}
& f(x, t)=Q_{F} \delta(x-c) \delta\left(t-t_{0}\right), \\
& H_{n}(t)=\left\{\begin{array}{cc}
0 & t<t_{0} \\
\frac{1}{\rho A} Q_{F} \frac{1}{\gamma_{n}^{2}} \frac{1}{\omega_{n}} & \\
\times \sin \left(\omega_{n}\left(t-t_{0}\right)\right) X_{n}(c) & t \geq t_{0}
\end{array}\right. \\
& w(x, t)=\left\{\begin{array}{cc}
0 & t<t_{0} \\
\frac{1}{\rho A} Q_{F} \sum_{n=1}^{\infty} \frac{1}{\gamma_{n}^{2}} \frac{1}{\omega_{n}} \sin \left(\omega_{n}\left(t-t_{0}\right)\right) \\
\times X_{n}(c) X_{n}(x) & t \geq t_{0}
\end{array}\right.
\end{aligned}
$$

- pulsed concentrated moment with constant amplitude acting at time $t_{0}$ 


$$
\begin{aligned}
& f(x, t)=Q_{M} \delta(x-d) \delta\left(t-t_{0}\right), \\
& H_{n}(t)= \begin{cases}0 & t<t_{0} \\
-\frac{1}{\rho A} Q_{M} \frac{1}{\gamma_{n}^{2}} \frac{1}{\omega_{n}} \sin \left(\omega_{n}\left(t-t_{0}\right)\right) & t \geq t_{0} \\
\times \frac{\mathrm{d}}{\mathrm{d} x} X_{n}(d) & t<t_{0}\end{cases} \\
& w(x, t)=\left\{\begin{array}{cc}
0 & \\
-\frac{1}{\rho A} Q_{M} \sum_{n=1}^{\infty} \frac{1}{\gamma_{n}^{2}} \frac{1}{\omega_{n}} \sin \left(\omega_{n}\left(t-t_{0}\right)\right) & \\
\times \frac{\mathrm{d}}{\mathrm{d} x} X_{n}(d) X_{n}(x) & t \geq t_{0}
\end{array}\right.
\end{aligned}
$$

\subsection{A numerical example}

As an example which shows the difference between results obtained just after action of excitation for the beam with zero initial condition (i.e $w_{0}(x)=0$ and $v_{0}=0$ in formulae (3)), based on the given formula (16) and formulae used for steady-state conditions, the vibration of a simply supported beam excited by harmonic change in time domain, concentrated force is considered. The force applied in the middle of a beam (26) can be expressed in the form

$$
f(x, t)=F_{1} \delta\left(x-\frac{1}{2}\right) \sin (\nu t) .
$$

The boundary conditions takes a form

$$
\left\{\begin{array}{l}
w(0, t)=0 \\
\frac{\partial^{2}}{\partial x^{2}} w(0, t)=0 \\
w(l, t)=0 \\
\frac{\partial^{2}}{\partial x^{2}} w(l, t)=0
\end{array} .\right.
$$

Hence the eigen-functions take the simplest form from those given in Table II $\left(X_{n}(x)=\sin \left(\lambda_{n} x\right), \lambda_{n}=\frac{n \pi}{1}\right.$, $\left.\omega_{n}=\frac{n^{2} \pi^{2}}{l^{2}} \sqrt{\frac{E I}{\rho A}}, n=1,2, \ldots,+\infty\right)$.

The amplitude of concentrated force is equal to $F_{1}=10 \mathrm{~N}$. The beam was made of steel $\left(E=2.1 \cdot 10^{11} \mathrm{~Pa}\right.$, $\left.\rho=7800 \mathrm{~kg} / \mathrm{m}^{3}\right)$ with length of $l=1 \mathrm{~m}$, and square cross-section $0.01 \times 0.01 \mathrm{~m}^{2}$. The angular frequency of excitation was $\nu=60 \mathrm{rad} / \mathrm{s}$. Material damping was neglected. The analytical solution has the form

$$
\begin{aligned}
& w(x, t)=\frac{2}{1} \frac{1}{\rho A} F_{1} \sum_{n=1}^{\infty} \frac{1}{\omega_{n}^{2}-\nu^{2}} \\
& \quad \times\left[\sin (\nu t)-\frac{\nu}{\omega_{n}} \sin \left(\omega_{n} t\right)\right] \sin \left(\lambda_{n} \frac{1}{2}\right) \sin \left(\lambda_{n} x\right) .
\end{aligned}
$$

In the analysis, the first five modes were considered.

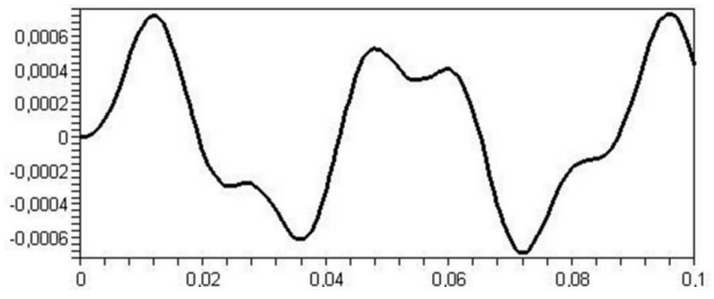

Fig. 1. Displacement in the middle of a beam - complete analytical solution.

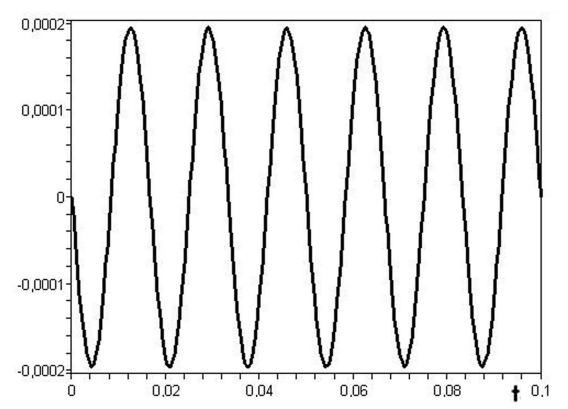

Fig. 2. Displacement in the middle of a beam steady-state analytical solution.

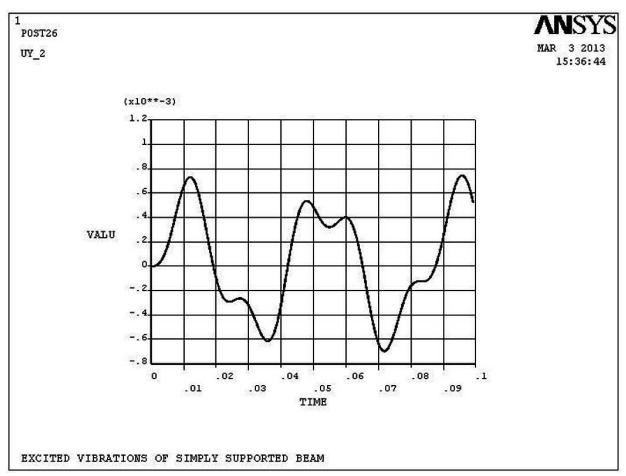

Fig. 3. Displacement in the middle of a beam - complete FEM solution.

Displacement of the middle of a beam just after beginning of vibrations is shown in Fig. 1. In Fig. 2, the solution for the steady-state case is shown. Important difference should be noted in values and form of the response. For realistic case, due to material damping, the solution shown in Fig.1 tends to solution shown in Fig. 2 for large values of time. For comparison, the result obtained based on the same assumptions by finite element method (FEM) solution (Ansys FEM package, transient dynamic analysis) is shown in Fig. 3. The solution is the same as for analytical result shown in Fig. 1 as the form and range of values is concerned.

\section{Conclusions}

Application of distributions allows for analytical formulation of the problem of excited vibrations of a beam loaded by concentrated forces and moments. The method gives a consistent formulation of the problem.

The analytical solution can be applied for verification of the solutions obtained by approximate approach e.g. by FEM.

Possibility of describing external load in the form of concentrated moments, allows for the analytic simulation of the reduction of vibrations of a beam by application of the piezoelectric elements, which are in practice the source of external moment type excitation put in relatively small area of the beam. 
To consider material damping in long-time analysis starting at the beginning of the vibrations, the analytical solution with damping should be applied (equation of motion of a beam with internal damping). For the analysis sufficiently late after start of vibrations, the steady-state solution can be applied.

\section{References}

[1] H. Marcinkowska, Distributions, Sobolev Spaces, Differential Equations, PWN, Warsaw 1993, (in Polish).

[2] S.O.R. Moheimani, A.J. Fleming, Piezoelectric Transducers for Vibration Control and Damping, Springer, London 2006.

[3] A. Brański, Acta Phys. Pol. A 121, A-126 (2012).

[4] A. Brański, G. Lipiński, Acta Phys. Pol. A 119, 936 (2011).

[5] M. Kozień, J. Wiciak, Acta Phys. Pol. A 116, 348 (2009).
[6] S. Kasprzyk, Acta Phys. Pol. A 119, 981 (2011).

[7] W. Łatas, P. Martynowicz, Engineering Modeling 44, 187 (2012), (in Polish).

[8] R. Łączkowski, Vibrations of Heat Turbines Elements, WNT, Warsaw 1974, (in Polish).

[9] S. Woroszył, Examples and Exercises on Theory of Vibrations, Part 2, Continuous Systems, PWN, Warsaw 1984, (in Polish).

[10] M. Kozień, J. Nizioł, Technical Trancactions 4-M, 1 (1993) (in Polish).

[11] A. Brański, M. Borkowski, S. Szela, Acta Phys. Pol. A 118, 17 (2010).

[12] A. Brański, S. Szela, Archiv. Acoust. 33, 521 (2008).

[13] M. Wiciak, Acta Phys. Pol. A 121, A-142 (2012).

[14] Z. Osiński, Theory of Vibrations, PWN, Warsaw 1980, (in Polish).

[15] J.W.S. Rayleigh, Theory of Sound, Dover Publications, New York 1945. 